Instability, Chaos and Predictability in Celestial Mechanics and Stellar Dynamics
Copyright 1992 by Nova Science Publishers, Inc. All rights of reproduction in any form reserved.

ISBN 1-56072-054-9

\title{
StABILITY OF SELF-GRAVITATING FINITE DISKS
}

\author{
Ashok Ambastha \\ Udaipur Solar Observatory, 11 Vidya Marg, Udaipur - 313001, India
}

\section{A BSTRACT}

Large scale, or "global" stability of finite radius, razor thin disk of self gravitating matter in presence of a massive halo surrounding the disk is studied as an eigen-value problem. Effect of the halo mass and size on the allowed global spiral eigenmodes of the disk is discussed.

\section{INTRODUCT ION}

The density wave theory has been one of the most successful approach to explain the stability and spiral structure of disk galaxies (Lin and Shu 1964; Lin, Yuan and Shu 1969). In the conventional 'local' theory, spiral perturbations of the form, $\tilde{\zeta}=\hat{\zeta} \exp \{i(\omega t+\mathbf{k r}+m \theta)\}$ are assumed to exist with the condition of tight winding, i.e., $|k| r \gg 1 ; k$ being the wave number $2 \pi / \lambda$, and a dispersion relation $D(k, \omega)=0$ is., obtained. Spiral patterns are termed as "leading" or "trail ing" according as $k>0$ or $k<0$. In the local analysis there remained several difficulties, namely, (i) its validity for tight spirals only, while in nature most galaxies exhibit rather open structures; (ii) existence of many free parameters; (iii) no distinction between the leading and trailing types of spiral patterns; and (iv) radial propagation of the spiral density waves (Toomre 1969). Additionally, since gravitational force is a long range one, a local analysis is not adequate especially for perturbations having wavelengths comparable to the characteristic dimensions of the system. Consequently, a global or large scale stability analysis of self-gravitating disks with appropriate boundary conditions is essential. 

FORMULAT ION

In what follows, we use a fluid dynamical description of model galaxy - in cylindrical coordinates, being the natural coordinate system for describing flat disk configurations:

$$
\begin{aligned}
& \frac{\partial \sigma}{\partial t}+\frac{1}{r} \frac{\partial}{\partial r}(r \sigma u)+\frac{1}{r} \frac{\partial}{\partial \theta}(\sigma v)=0 \\
& \frac{\partial u}{\partial t}+u \frac{\partial u}{\partial r}+\frac{v}{r} \frac{\partial u}{\partial \theta}-\frac{v^{2}}{r}=-\frac{1}{\sigma} \frac{\partial p}{\partial r}+\frac{\partial \psi}{\partial r} \\
& \frac{\partial v}{\partial t}+u \frac{\partial v}{\partial r}+\frac{v}{r} \frac{\partial v}{\partial \theta}+\frac{u v}{r}=-\frac{1}{r \sigma} \frac{\partial p}{\partial \theta}+\frac{1}{r} \frac{\partial \psi}{\partial 0}
\end{aligned}
$$

where $\sigma(r, \theta, t), p(r, \theta, t), u(r, \theta, t), v(r, \theta, t)$ and $\psi(r, \theta, t)$ are surface density, thermal pressure, radial and azimuthal velocities, and net gravitational potential, respectively. Self-gravitational potential $\psi$ due to surface density distribution of matter, $\sigma$, on the flat disk is given by the Poisson's equation:

$$
\nabla^{2} \psi=-4 \pi G \delta(z) \sigma
$$

In order to close the system of equations, we use an equation of state between pressure and density:

$$
p=c \sigma^{\gamma}
$$

Let us consider the equilibrium state, i.e., time independent, axisymmetric disk having surface density $\Sigma(r)$, and thermal pressure $P(r)$ with no radial flows, i.e., $U(r)=0$. The azimuthal velocity, $V(r)=r \Omega^{2} ; \Omega(r)$ being $t$ he angular velocity, is then obtained from the radial component of $t$ he momentum conservation equation ( 2 ):

$$
-\frac{\mathrm{v}^{2}}{\mathrm{r}}=-\frac{1}{\Sigma} \frac{\mathrm{d} \mathrm{P}}{\mathrm{dr}}+\frac{\mathrm{d} \Psi}{\mathrm{dr}}
$$

where $\Psi(r)$ is the net gravitational potential at a point $r$ in the plane of the disk, and is given by:

$$
\Psi(r)=\Psi_{d}(r, z=0)+\Psi_{b}(r)+\Psi_{h}(r)
$$

where $\Psi_{d}$ is self-consistent gravitational potential of the disk, and $\Psi_{b}, \Psi_{h}$ are the external or fixed potential contributions of the central bulge and the halo components, respectively. 
It is possible to obtain gravitational potential associated with a general surface density distribution of the form (cf. C1utton-Brock 1972):

$$
\sigma(r, \theta, t)=\sum_{m=0}^{\infty} \sigma_{m}(r) \exp [i(\omega t+m \theta)]
$$

Corresponding gravitational potential is then

$$
\psi(r, \theta, t, z)=\sum_{m=0}^{\infty} \psi_{m}(r, z) \exp [i(\omega t+m \theta)]
$$

where

$$
\psi_{m}(r, z)=2 \pi G \int_{0}^{\infty} A_{m}(x) J_{m}(x r) \exp (-x|z|) d x
$$

$A_{m}(x)$ being the fankel transform of $\sigma_{m}(r)$, such that

$$
A_{m}(x)=\int_{0}^{\infty} \sigma_{m}(r) J_{m}(x r) r d r .
$$

For a generalized density distribution, expanded in terms of the Bessel's functions:

$$
\begin{array}{rlrl}
\sigma_{m}(r) & =\sum_{j=0}^{\infty} a_{j m} J_{m}\left(\lambda_{j n} r\right) ; & r \leq R_{d} \\
& =0 & ; & r>R_{d}
\end{array}
$$

one has

$$
\begin{aligned}
\psi_{m}(r)= & 2 G \sum_{j=0}^{\infty} a_{j m}\left(\frac{J_{m}\left(\lambda_{j m} r\right)}{\lambda_{j m}}+\frac{4}{\pi^{2} Y_{m}\left(\lambda_{j m} R\right)}\right. \\
& \left.\times \int_{0}^{\infty} \frac{I_{m}\left(k^{\prime} r\right) K_{m}\left(k^{\prime} r\right)}{\lambda_{m}^{2}+k^{\prime 2}} d k^{\prime}\right) ; r \leq R \\
= & 2 \pi G \sum_{j=0}^{\infty} a_{j m}\left(\frac{4}{\pi^{2} Y_{m}\left(\lambda_{j m} R\right)} \int_{0}^{\infty} \frac{I_{m}\left(k^{\prime} R\right) K_{m}\left(k^{\prime} r\right)}{\lambda_{j m}^{2}+k^{\prime 2}} d k^{\prime}\right) ; r>R
\end{aligned}
$$

where $\lambda_{j}$ is the $j t h$ root of $J_{m}(\lambda R)=0$ (Yabushita 1969).

Now, let us consider stability of the disk against infinitesimally small perturbations, $\tilde{\zeta}(r, \theta, t)=\hat{\zeta}(r) \exp \{i(\omega t+m \theta)\}$, 
where $\hat{\zeta}, \omega \equiv\left(\omega_{r}, \omega_{i}\right), m$ and $\Omega_{p}=-w_{r} / m$ are the amplitude,frequency, wave-number and the pattern-velocity of the perturbation, respectively. The perturbation grows or damps exponentially, or remains oscillatory according as $\omega_{i}<0,>0$ or $=0$. Radial parts of the perturbations are expanded as follows (cf. Ambast ha and Verma, 1983)

$$
\begin{aligned}
& \hat{\sigma}(r)=\sum_{k=0}^{\infty} C_{k} J_{m}\left(\lambda_{k} r\right) ; \quad \hat{\psi}(r)=\sum_{k=0}^{\infty} C_{k} \psi_{m}(r) \\
& \hat{u}(r)=1 / 2 \sum_{k=0}^{\infty}\left[A_{k} J_{m+1}\left(\lambda_{k} r\right)+B_{k} J_{m-1}\left(\lambda_{k} r\right)\right] \\
& \hat{v}(r)=1 / 2 \sum_{k=0}^{\infty}\left[A_{k} J_{m+1}\left(\lambda_{k} r\right)-B_{k} J_{m-1}\left(\lambda_{k} r\right)\right]
\end{aligned}
$$

These expansions are substituted in equations (1)-(4) after 1 inearization; then multiplied by suitable orthogonal functions and integrated over the plane of the disk in the interval $0 \leq r \leq 1$. As a result, an infinite set of algebraic equations is derived which could more conveniently be expressed in the form of an eigenvalue problem for the perturbations:

$$
\mathrm{MZ}=\omega \mathrm{Z}
$$

where $M$ is a $30 \times 3 \infty$ matrix, non-symmetric in general and $\mathrm{Z}$ is a column matrix constituted by the basis vectors $\hat{\sigma}(r), \hat{u}(r)$, $\hat{v}(r)$. In general, the eigenvalue problem expressed in equat ion (13) cannot be solved analytically, hence, we have used a numerical method involving elementary similarity transformation of a suitably truncated matrix in to evaluate the eigenvalues $\omega$ and the corresponding eigenvectors $Z$.

\section{GLOBAL STABILITY OF ONE COMPONENT DISKS}

Self-consistent global stability analysis has been earlier carried out by a number of workers for one-component,differentially rotating, finite radius flat disks models(Hunter 1963, 1965; Bardeen 1975; Iye 1978; Aoki et al. 1979; Pannatoni and Lau 1979; Ambastha and Varma 1983). In principle,the eigen-value problem given by equation (13) admits an infinite, discrete set of eigen frequencies comprising of both real and complex-conjugate pairs. Large scale smooth spiral patterns have been found associated with many of the complex eigenmodes, i.e., spirals appear as self-excited, normal modes of oscillations in the plane of flat, differentially rotating disks. Stability of both "cold" as well as "hot" self-gravitating disks have been examined against axisymmetric and nonaxisymmetric perturbations with no restriction imposed on radial wavelengt $h \lambda(r)$. 
In "cold" or pressure-less disk models, spiral modes with relatively smaller radial wavelengths are found to have much larger growth-rates as compared to their pattern frequencies. In other words, these modes with tight spiral arms are "explosively" unstable in cold disk models. As central condensation of the surface density is increased, growth-rates of "explosive" modes are found to increase further. On the contrary, these "explosive" modes become less violent when thermal energy or "hotness" of the disk is enhanced instead of the central condensation. Also, it is found that modes with tighter spiral patterns are stabilized faster than the modes having rather open spiral structures. However, large thermal energies are required in order to completely suppress all "explosive" modes. It is al so noticed that modes with regular tightly wrapped spiral patterns become irregular as "hotness" of $t$ he disk is increased. On the other hand, modes having rather open "leading" spiral patterns are gradually turned into smooth trailing patterns above a critical value of the thermal energy. Of course, eventually no unstable modes survived, i.e., no spiral structures is allowed, when thermal energy of the disk is made sufficiently large (Ambast ha and Varma 1983 ).

\section{MULTI-COMPONENT SP IRAL GALAXY MODELS}

It is well known that disk galaxies are complex a stronomical objects consisting of several components with widely varying properties, such as, (i) a flat rotating disk comprising of gas, dust, population I and II stars; (ii) central spheroidal bulge which is made up mainly of population I I objects; and (iii) a large "invisible" halo surrounding the disk and the bulge. Although the visible spiral structures of a galaxy are characteristic feature displayed by the gaseous and young stellar population of the flat disk component, their physical appearance seems to be governed by the size and massiveness of the central bulge, which does not exhibit spiral features itself (Freeman 1970). Apart from ignoring the major components of real galactic systems, viz., central spheroidal "bulge" and a "halo" around the disk, the one component disk galaxy models suffer from the presence of explosivel y unstable modes, and require considerably large thermal energies in order to suppress these violent instabilities. It has been earlier found by numerical simulations that inclusion of a large halo enveloping the disk suppresses explosive modes ( $H \circ \mathrm{hl}$ 1976; Berman, Brownrigg and Hockney 1978). In view of this, we have made a study on the large scale stability and structure of finite disk including various aspects of galactic composition. Ambast ha and Varma (1982) have earlier studied stability and spiral structures of a flat selfgravitating disk under the influence of an external gravitational force exerted by a fixed central bulge component. They have examined properties of the global eigenmodes for various ratios of the disk-to-bulge mass and radius. 
A massive halo, surrounding both the galactic disk and the central bulge may al so have a major influence on the dynamics and structures of the flat disk component. Presence of a massive halo around our own Galaxy, the Milky Way, is evidenced from the nearly flat rotational curve at large galacto-centric distances (Rubin, Ford and Thonard 1978; Bosma 1978; Krumm and Salpeter 1979). It has been inferred that the halo component of a galaxy could be around ten to twenty times as massive as the disk (Bok 1981).

In order to examine the influence of the halo component on the dynamics and structure of the disk component, we consider a razor thin disk with radius $R_{d}$ of self-gravitating matter (gas, dust and population I objects) having a surface density distribution $\sigma_{\mathrm{d}}(\mathbf{r})$ :

$$
\begin{aligned}
\sigma_{d}(r) & =\sigma_{0} J_{2}\left(\lambda_{0} r\right) ; & & \leq R_{d} \\
& =0 ; & r & >R_{d}
\end{aligned}
$$

where $\sigma_{O}$ is a constant and $R_{d}$ is radius of the disk. Further, we consider a spherical halo surrounding the disk having a volume density given by:

$$
\begin{array}{rlrl}
\rho_{h}(r) & =\rho_{o}\left[1+\left(r / R_{h}\right)^{2}\right]^{-1} & ; r \leq R_{h} \\
& =0 & ; r & >R_{h}
\end{array}
$$

where $\rho_{0}$ is the central density and $R_{h}$ is the core-radius of the halo. The corresponding gravitational potential due to the "fixed" halo mass is:

$\left.\Psi_{h}(r)=-2 \pi G \rho_{d} R_{h}^{2} \ln \left\{1+\left(r / R_{h}\right)^{2}\right\}+2\left(R_{h} / t\right) \tan ^{-1}\left(r / R_{h}\right)-2\right]$

Pe define here $\delta_{h}=M_{h}\left(R_{d}\right) /\left(M_{d}+M_{h}\right)$ or $\delta=M_{d} / M_{h}$ and $\eta=R_{h} /$ $R_{d}$ which determine ratios of masses and sizes of the disk and the halo.

Our main interest here is to understand influence of a halo on the large scale stability of the self-gravitating disk, hence for simplification we assume a 'cold' or pressureless disk. Effect of thermal pressure on the global modes has been investigated earlier (Ambastha and Varma 1983, Aoki et al. 1979). By carrying out a normal mode analysis described earlier, we $f$ ind that a number of unstable modes are allowed as in previous investigations. Unstable eigenmodes of the disk in presence of fixed halo potential for various values of $\delta$ keeping $n$ constant are shown in Figure (1). One finds that some of the eigenmodes are still "explosive", as in one- 

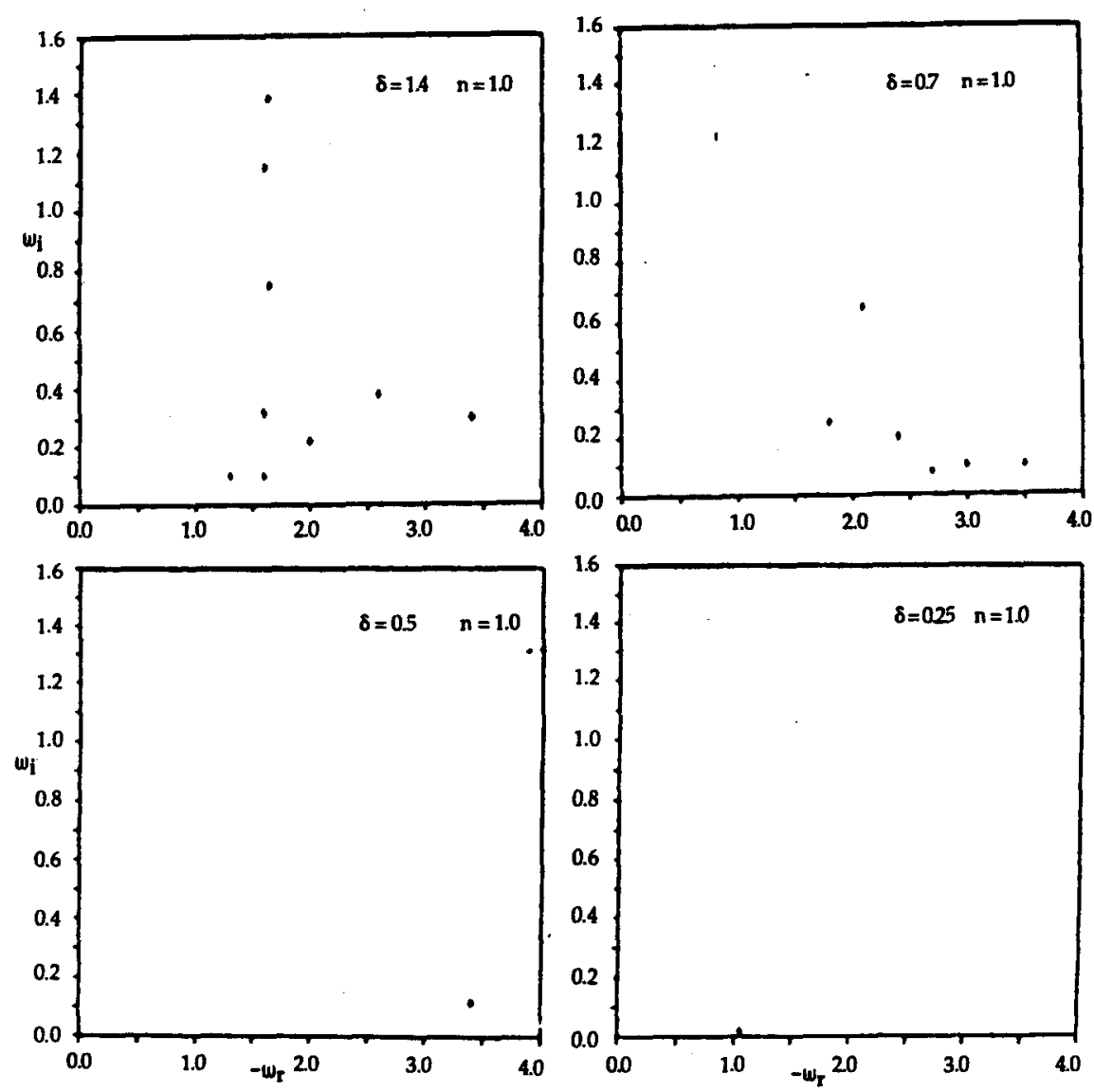

Figure 1: Growth-rates $\omega_{i}$ are plotted against frequency $\omega_{r}$ for the unstable eigenmodes of the disk as the disk-to-halo mass ratio, $\delta$ is decreased. The coreradius of the halo, is kept the same.

component "cold" disks, when the halo is not sufficiently massive as compared to the disk. However, when the halo mass is increased to around 4-5 times the disk mass, almost no $u n-$ stable modes are allowed, i.e., no large scale or "global" spiral patterns are permissible.

Figure 2 shows the unstable eigenmodes for a fixed diskto-halo mass ratio, $\delta=0.25$, but increasing values of coreradius of the halo component. One finds here that for a halo around 4 times more massive than the disk, no explosive modes 
allowed even when the core radius of halo is zero. Moreover, as the core-radius of the halo is increased, many of the unstable modes got stablized and eventualiy only a single unstable mode survived for $n>1$.
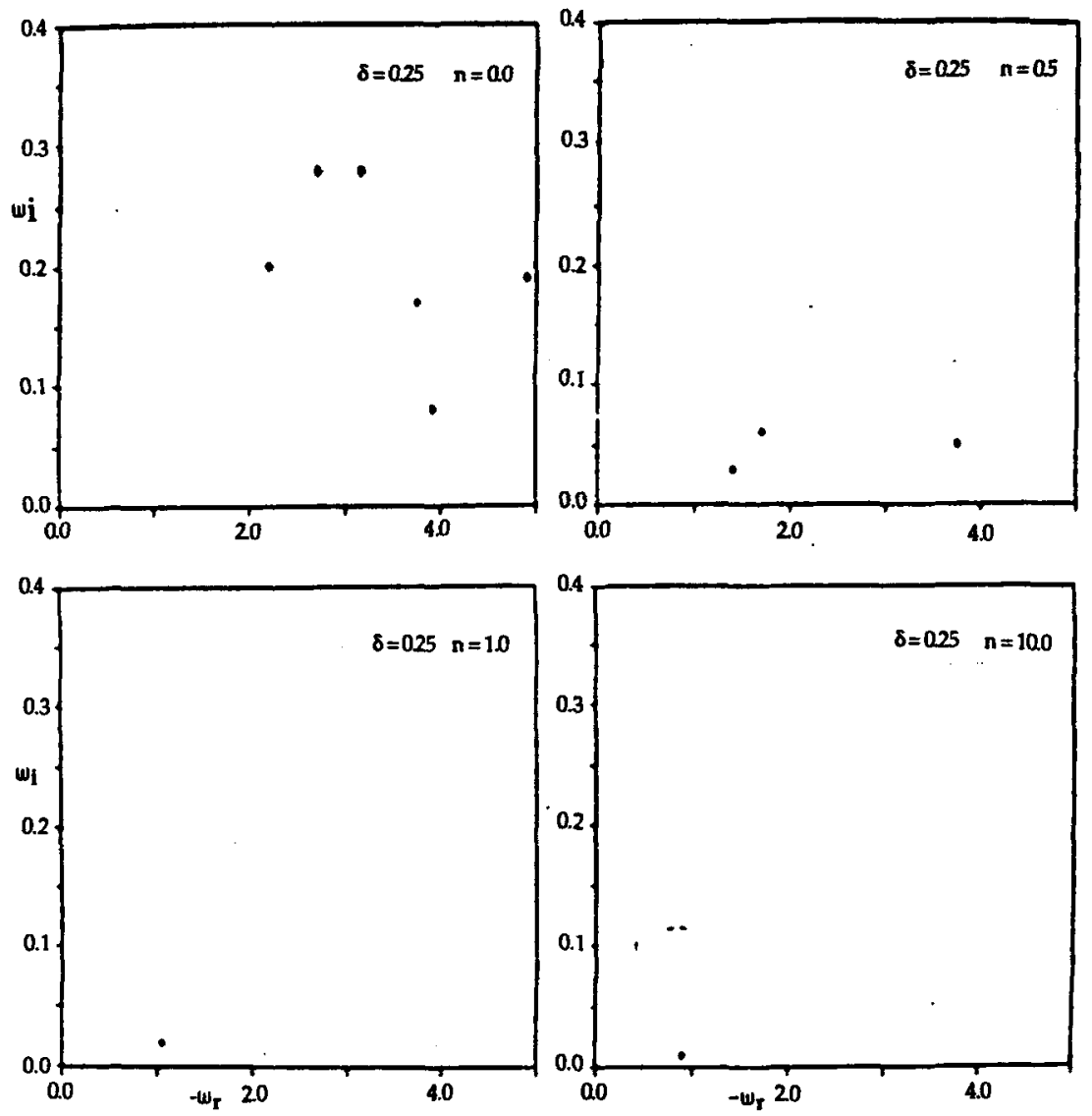

Tigure 2: Growth-rates $\omega_{i}$ against frequency $\omega_{r}$ for unstable eigenmodes of the disk as the ratio of the coreradius of the halo to the radius of the disk is increased. The halo mass is four times the disk mass.

Figure 3 shows eigen-patterns associated with some unstable modes for $\delta_{n}=0.9$ and $n=0.5$ and 1.0 . In the first case, there is an irregular spiral structure with rapid variation in amplitude along the spiral. However, smooth spiral structure is obtained when the halo core-radius is equal to or greater than the disk radius (see also Figure 4). 
$(-1.28,-0.82)$

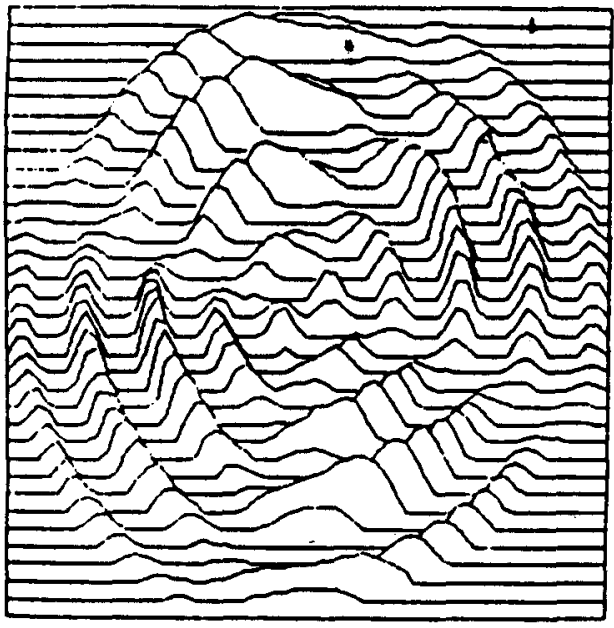

$n=0.5 \quad \delta_{n}=0.9$
$(-0.89,-0.54)$

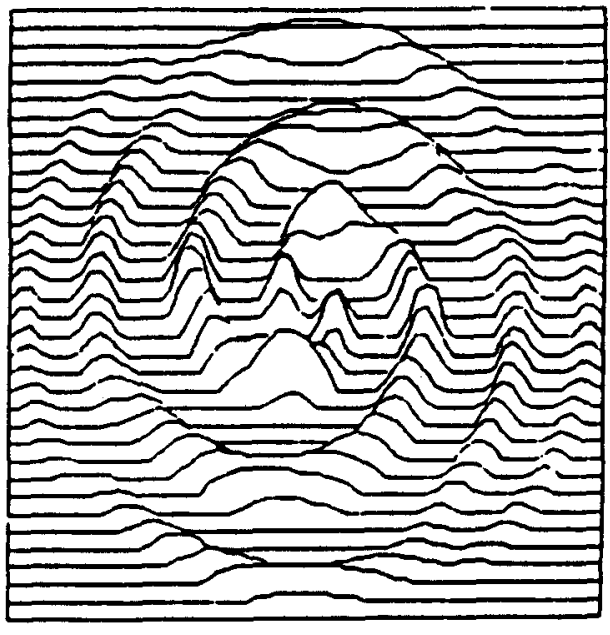

$n=1.0 \quad \delta_{n}=0.9$

Figure 3: Eigen-patterns associated with bi-symmetric $(m=2)$ surface density perturbations for selected eigenmodes. The halo mass is nine times the disk mask $n=0.5$ and 1.0 .

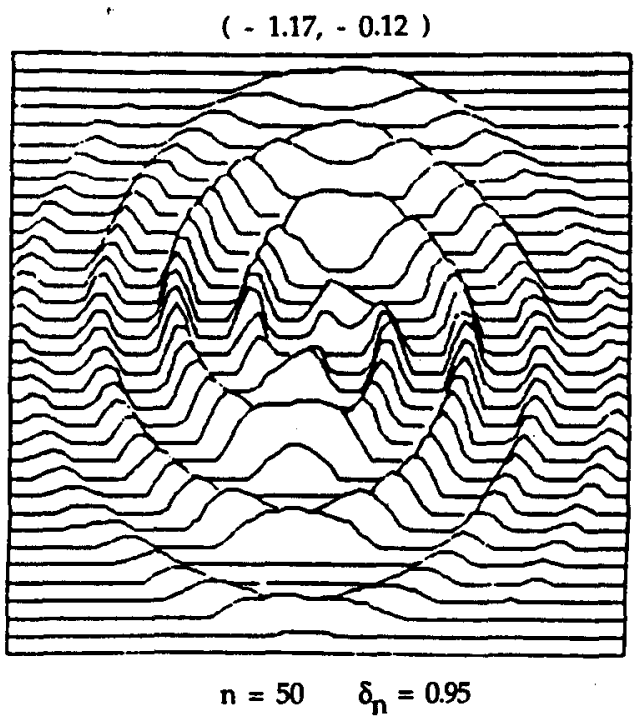

Tigure 4: Eigen-patterns associated with bi-symmetric $(m=2)$ curface density perturbation for the unstable mode at $n=50$ and $\delta_{n}=0.95$. 


\section{CONCLU SION}

Earlier studies have shown that unreasonably large thermal pressures, such that the thermal energy of the disk is comparable to its rotational energy, are required in order to stablize the disk against all unstable global modes (Ostriker and Peebles 1973, Ambastha and Varma 1983). Further, it is rather difficult to construct kinematically stable disk models with a reasonable pressure distribution when there is no halo present. Global modes of oscillations tend to grow explosively in a model disk galaxies in a few rotational periods unless there exists a massive halo. On the other hand, it is found here that very large and massive halo tends to prohibit spiral structures in the disk. It is also found that while cold disks without halo mostly allowed "leading" spiral patterns, smooth "trailing" patterns are allowed when there is a halo around the cold disk.

\section{REF ER ENC ES}

[1] Ambastha, A. and Varma, R.K. (1982), J.A strophys.Astron. 3,125 .

[ 2 ] Ambastha, A. and Varma, R.K. (1983), Astrophys.J.264,413.

[3] Aoki, S., Noguchi, M. and Iye, M. (1979), Publ .Astron. Soc. Japan 31,737.

[4] Bardeen, J.(1975), in IAU Symposium No.69: Dynamics of Stellar Systems, Ed.A.Hayli, D.Reidel, Dordrec ht,p.297.

[5] Berman, R.H., Brownrigg, D.R.K. and Hockney, R.W.(1978), Mon. Not. Royal Astron. Soc. 185,861.

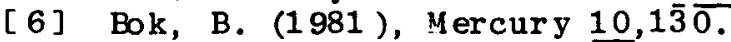

[7] Bosma, A. (1978), Ph.D. Thesis, Rijksuniv., Groningen.

[8] Clutton-Brock, M. (1972), Astrophys. Space Sci. 16,101.

[9] Freeman, K.C. (1970), A strophys. J.160, 811.

[10] Hohl, F.H. (1976), Astron. J.81, 30 .

[11] Hunter, C. (1963), Mon. Not.Royal Astron. Soc. 126, 299.

[12] Hunter, C. (1965 3 , Mor. Not.Royal Astron. Soc. 129, 321.

[13] Iye, M. (1978), Publ . Astron. Soc. Japan 30, 223.

[11] Krumm, N. and Salpeter, E.E. (1979), A strōn.J. 84,1138.

[15] Lin, C.C. and Shu, F.H. (1964), A strophys. J. 140, 646.

[16] Lin, C.C., Yuan, C. and Sha, F.H. (1969), Astrophys. J. 155,721 .

[17] Ostriker, J.P. and Peebles, P.J.E. (1973), Astrophys.J . 186,467 .

[18] Pannatoni, R.F. and Lau, Y.Y. (1979), Proc. Nat.Acad. Sci. 76,4 .

[19] Rubin, V.C., Ford, W.K. and Thonard, N. (1978), Astrophys. J. 225, L107.

[ 20] Toomre, A. (1969), A strophys. J .158, 899 .

[ 21 ] Yabushita, S. (1969), Mon. Not.Royal Astron. Soc. 142, 201. 